\title{
FAULT DIAGNOSIS OF ROTARY MACHINE BY FUZZY NEURAL NETWORKS
}

\author{
Sheng Zhang*, Toshiyuki Asakura*, Xiaoli Xu**, Baojie Xu** \\ *Dept. of Intelligence, Faculty of Engineering, Fukui University, zhangsheng@scmc.mech.fukui-u.ac.jp. \\ **Department of Mechanical Engineering, Beijing Institute of Machinery, xuxiaoli@cenpork.com
}

\begin{abstract}
This paper is concerned with a research about fuzzy neural networks and application in fault diagnosis of rotary machine. To build robust fault diagnosis by neural networks, fuzzy neural networks are proposed. Fuzzy neural networks can memorize fault patterns and recognize fault patterns by association, and have good tolerance to unstable practical sample data. Through application in monitoring and fault diagnosis of pump sets of oil plants ${ }^{[1]}$, it was verified that fuzzy neural networks are effective to handle practical sample data and make accurate fault diagnosis.
\end{abstract}

\section{INTRODUCTION}

Rotary machines such as generators, electrical motors and civil engines are widespread used in every aspects of our society. They are very important because they provide power to run our society. To keep these rotary machines in good working conditions and avoid losses caused by unexpected accidents, fault diagnosis systems are necessary, especially the automatic fault diagnosis systems, for at present more and more plants have been automated. In some cases such as in nuclear generation stations, the fault parts are dangerous for human beings to access, the automatic fault diagnosis system are in urgent demand.

Fuzzy neural networks for fault diagnosis are optimized fuzzy associate memories. Fuzzy neural networks can memorize state patterns and associate, actually by matching sample pattern and the stored standard patterns to make a fault diagnosis.

In this paper, at first the structure of fuzzy neural networks for fault diagnosis is discussed. A new train algorithm is proposed. The algorithm is based on fuzzy differential rules deduced from Hebb synthesis calculation. Mathematic verification about the algorithm of training is given. Second, the application of fault diagnosis systby fuzzy neural networks on pump sets of oil plants is introduced.
Finally practical cases are used to verify that fuzzy neural networks are effective to handle practical sample data and make accurate fault diagnosis.

\section{FUZZY NEURAL NETWORKS FOR FAULT DIAGNOSIS}

\subsection{Why fuzzy neural networks ${ }^{[2,3,4,5]}$}

With the modern equipment becoming more and more complex and the working conditions becoming very serious, the non-stationary and non-linear characters become more and more remarkable. So the vibration signals become complex which made the monitoring of the sets working conditions and fault diagnosis more difficult than before. Traditional neural networks cannot be suitable any longer. For example, back propagation neural networks were used to monitoring and make fault diagnosis of water pump sets. Because the practical sample data change in a big scale range, the fault diagnosis system by back propagation neural networks often reports uncertain diagnosis results and its diagnosis result become very unreliable. To solve this difficulty, we try to use fuzzy logic to improve the traditional neural networks. Unlike traditional neural networks, fuzzy neural networks adopt fuzzy logic, like humans do. Therefore fuzzy neural networks have good tolerance to practical sample data and can work much more reliable than traditional neural networks.

\subsection{The fuzzy neural cell}

In this research, two kinds of fuzzy neural cells are used. They are fuzzy cell and fuzzy logic cell. Fuzzy cell is showed as Fig. 1:

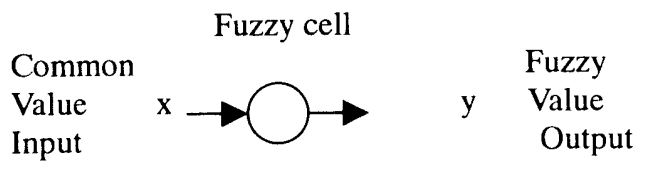

Fig. 1 Fuzzy cell

Fuzzy cell converts common value input into fuzzy value output. The relation between input and output is 
described as function of equation (1).

$$
y=F(x)
$$

In this research function $F$ adopts five-phase function $^{[6,7,8]}$ as Fig. 2 shows. Here input and output are all normalized to numbers from 0 to 1 .

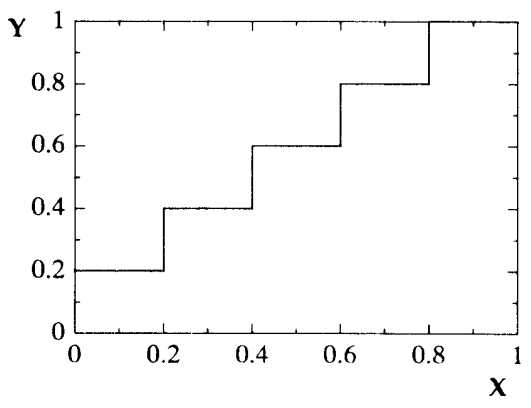

Fig. 2 Five-phase function of fuzzy cell Fuzzy function really reflects the thinking habit of human beings. When human beings think, they usually use rough estimation instead of accurate measurement. Especially in practical fault diagnosis in the plants.

Fuzzy logic cell is showed as Fig. 3. This is multiple input mono output neural cell. As for every input[i], there is a corresponding weight[i].

Fuzzy logic cell

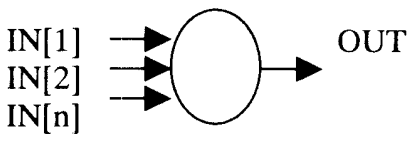

Fig. 3 Fuzzy logic cell

Relation between input and output follows equation (2). Here weights, inputs and outputs are all normalized to numbers from 0 to 1 .

$$
\text { out }=\max \{\min (\operatorname{in}[i], \text { weight }[i])\}
$$

Equation (2) is called as fuzzy logic function. It is also called max-min principle ${ }^{[9,10,11]}$. It is can be explained that the inputs follow competitive rule and outputs follow max rule. That is to say, the inputs are competitive between themselves, so the influence of every input on the output is decreased. On the other hand, the output is decided by the max input, the output is only related to the max input and other inputs are omitted. This is the key idea of fuzzy logic cell. It reflects the deduction and judge process of human beings.

2.3 Structure of fuzzy neural networks.

Fig. 4 is the structure of fuzzy neural networks. Every layer has six neural cells. The fuzzy layer is to fuzzy the input sample data. The associative layer is consisted of the fuzzy logical cells which can memorize and associate standard fault patterns.

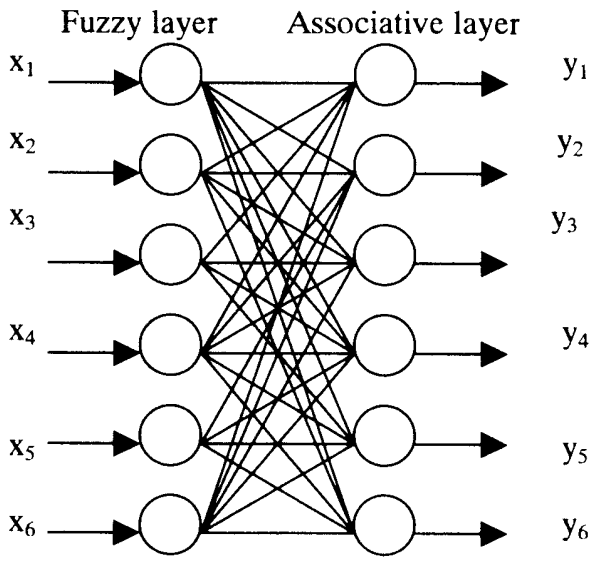

Fig. 4 Structure of fuzzy neural network

The neural networks showed in Fig. 4 are called fuzzy associative memory (FAM for brief). Researchers have proved that the neural networks can memorize and recall patterns by association.

Suppose there is input-output pair like equation (3) shows:

$$
\begin{aligned}
& X=\left[\mathrm{x}_{1}, \mathrm{x}_{2}, \mathrm{x}_{3}, \mathrm{x}_{4}, \mathrm{x}_{5}, \mathrm{x}_{6}\right] \\
& =[0.8,0.2,0.2,0.2,0.2,0.2] \\
& Y=\left[\mathrm{y}_{1}, \mathrm{y}_{2}, \mathrm{y}_{3}, \mathrm{y}_{4}, \mathrm{y}_{5}, \mathrm{y}_{6}\right] \\
& =[0.2,0.2,0.2,0.8,0.2,0.2]
\end{aligned}
$$

Then according to equation (4) calculate weights. Equation (4) is often used to initial weight.

$$
\text { Weight }[i][j]=\min \left(x_{i}, y_{j}\right)
$$

For the pattern pair of equation (3), a weight matrix as Table. 1 shows is obtained:

Table. 1 Weight matrix for sample pair X Y
\begin{tabular}{|l|l|l|l|l|l|l|}
\hline $\mathrm{W}_{\text {ii }}$ & 1 & 2 & 3 & 4 & 5 & 6 \\
\hline 1 & 0.2 & 0.2 & 0.2 & 0.8 & 0.2 & 0.2 \\
\hline 2 & 0.2 & 0.2 & 0.2 & 0.2 & 0.2 & 0.2 \\
\hline 3 & 0.2 & 0.2 & 0.2 & 0.2 & 0.2 & 0.2 \\
\hline 4 & 0.2 & 0.2 & 0.2 & 0.2 & 0.2 & 0.2 \\
\hline 5 & 0.2 & 0.2 & 0.2 & 0.2 & 0.2 & 0.2 \\
\hline 6 & 0.2 & 0.2 & 0.2 & 0.2 & 0.2 & 0.2 \\
\hline
\end{tabular}

Now according to equation (2) to associate, input sample $\mathrm{X}$ of equation (3), the result of association is :

$$
Z=[0.2,0.2,0.2,0.8,0.2,0.2]
$$


$\mathrm{Z}$ of equation (5) is completely in accordance with $\mathrm{Y}$ in equation (3), this verifies the fuzzy neural networks as Fig. 4 can memory pattern pair and associate.

In fact, the fuzzy neural networks above can memory any patterns. The problem is that the memory capacity of the fuzzy neural networks is limited. To make use of the fuzzy neural networks in practical situation, the algorithm of training needs improvement.

\subsection{Improvement of training algorithms ${ }^{[12,13,14]}$}

Ordinary fuzzy associative memories can only memorize one pattern, so its application is very limited. The optimized fuzzy associative memories adopt a new training method to adjust weights, which is based on fuzzy differential rules deduced from Hebb synthesis calculation. This training process takes energy function as the standard of the neural networks, adjusting weights according to the slope down rule. The training steps are described as follows:

(1) To initial weights, study times and error limit.

(2) To adjust weights as follows:

$$
\begin{aligned}
& W_{i j}^{(t+1)}=W_{i j}^{(t)}-\alpha(t) \frac{\partial E_{k}}{\partial W_{i j}} \\
& \frac{\partial E_{k}}{\partial W_{i j}^{(t)}}=\left[{\underset{r=1}{\vee}}_{r=1}^{N}\left(x_{r}^{(k)} \wedge w_{r j}^{(t)}\right)-y_{j}\right] \delta_{i j}^{(t)} \\
& \delta_{i j}^{(t)}=\frac{\partial}{\partial W_{i j}^{(t)}}\left(x_{r}^{(k)} \wedge w_{r j}^{(t)}-y_{j}\right) \\
& =\left\{\begin{array}{cc}
1 & \max \left(x_{r}^{(k)} \wedge w_{r j}^{(t)}\right) \\
0 & \text { ifnot }
\end{array}\right.
\end{aligned}
$$

(3) To calculate errors.

(4) To return to step 2 .

\subsection{Mathematical verification of the algorithms.}

The mathematical verification of the algorithms is demonstrated as following:

(1) Energy Function

$$
E_{k}=\sum\left[\vee\left(x_{r}^{(k)} \wedge w_{r j}^{(t)}\right)-y_{j}\right]^{2} \geq 0
$$

(2) Stability of the network

$$
\begin{array}{r}
\frac{\partial E_{k}}{\partial t}=\frac{\partial E_{k}}{\partial W_{i j}^{(t)}}\left(W_{i j}^{(t+1)}-W_{i j}^{(t)}\right) \\
=-\alpha(t)\left(\frac{\partial E_{k}}{\partial W_{i j}^{(t)}}\right)^{2} \leq 0
\end{array}
$$

(3) The existence of minimum value

$$
\begin{aligned}
\frac{\partial^{2} E_{k}}{\partial W_{i j}^{(t)}} & \left.=\left\{\underset{r=1}{N}\left(x_{r}^{(k)} \wedge w_{r j}^{(t)}\right)-y_{j}\right] \delta_{i j}^{(t)}\right\} / \partial W_{i j}^{(t)} \\
& =\left(\delta_{i j}^{(t)}\right)^{2} \geq 0
\end{aligned}
$$

According to Lyapurov theorem, the network has stable and minimum weight vectors.

\subsection{Training and association}

Computer program is made for the training algorithms to test the memory and association ability of the fuzzy neural networks with new training algorithms. The input standard patterns vectors are listed in Table.2, the corresponding output are listed in Table. 3.

Table. 2 Input pattern vectors

\begin{tabular}{|c|c|c|c|c|c|}
\hline $\mathrm{X}_{1}$ & $\mathrm{X}_{2}$ & $\mathrm{X}_{3}$ & $\mathrm{X}_{4}$ & $\mathrm{X}_{5}$ & $\mathrm{X}_{6}$ \\
\hline 0.8 & 0.2 & 0.2 & 0.2 & 0.2 & 0.2 \\
\hline 0.2 & 0.2 & 0.8 & 0.2 & 0.2 & 0.2 \\
\hline 0.2 & 0.8 & 0.2 & 0.2 & 0.2 & 0.2 \\
\hline 0.2 & 0.2 & 0.2 & 0.8 & 0.2 & 0.2 \\
\hline 0.2 & 0.2 & 0.2. & 0.2 & 0.2 & 0.8 \\
\hline 0.2 & 0.2 & 0.2 & 0.2 & 0.8 & 0.2 \\
\hline
\end{tabular}

Table. 3 Output vectors

\begin{tabular}{|l|l|l|l|l|l|l|}
\hline $\mathrm{Y}_{1}$ & 0.2 & 0.2 & 0.2 & 0.2 & 0.2 & 0.8 \\
\hline $\mathrm{Y}_{2}$ & 0.2 & 0.2 & 0.2 & 0.2 & 0.8 & 0.2 \\
\hline $\mathrm{Y}_{3}$ & 0.2 & 0.2 & 0.2 & 0.8 & 0.2 & 0.2 \\
\hline $\mathrm{Y}_{4}$ & 0.2 & 0.2 & 0.8 & 0.2 & 0.2 & 0.2 \\
\hline $\mathrm{Y}_{5}$ & 0.2 & 0.8 & 0.2 & 0.2 & 0.2 & 0.2 \\
\hline $\mathrm{Y}_{6}$ & 0.8 & 0.2 & 0.2 & 0.2 & 0.2 & 0.2 \\
\hline
\end{tabular}

Table. 4 Weight matrix after training

\begin{tabular}{|l|l|l|l|l|r|r|}
\hline $\mathrm{W}_{\mathrm{ij}}$ & 1 & 2 & 3 & 4 & 5 & 6 \\
\hline 1 & 0.2 & 0.2 & 0.2 & 0.2 & 0.2 & 0.8 \\
\hline 2 & 0.2 & 0.2 & 0.2 & 0.8 & 0.2 & 0.2 \\
\hline 3 & 0.2 & 0.2 & 0.2 & 0.2 & 0.8 & 0.2 \\
\hline 4 & 0.2 & 0.2 & 0.8 & 0.2 & 0.2 & 0.2 \\
\hline 5 & 0.8 & 0.2 & 0.2 & 0.2 & 0.2 & 0.2 \\
\hline 6 & 0.2 & 0.8 & 0.2 & 0.2 & 0.2 & 0.2 \\
\hline
\end{tabular}

To test trained neural networks, input patterns of Table. 2 , the result of association is listed in Table. 5 .

Table. 5 Result of associate 


\begin{tabular}{|l|l|l|l|l|l|l|}
\hline $\mathrm{Z}_{1}$ & 0.2 & 0.2 & 0.2 & 0.2 & 0.2 & 0.8 \\
\hline $\mathrm{Z}_{2}$ & 0.2 & 0.2 & 0.2 & 0.2 & 0.8 & 0.2 \\
\hline $\mathrm{Z}_{3}$ & 0.2 & 0.2 & 0.2 & $0 . .8$ & 0.2 & 0.2 \\
\hline $\mathrm{Z}_{4}$ & 0.2 & 0.2 & 0.8 & 0.2 & 0.2 & 0.2 \\
\hline $\mathrm{Z}_{5}$ & 0.2 & 0.8 & 0.2 & 0.2 & 0.2 & 0.2 \\
\hline $\mathrm{Z}_{6}$ & 0.8 & 0.2 & 0.2 & 0.2 & 0.2 & 0.2 \\
\hline
\end{tabular}

Table. 5 shows the improved fuzzy neural networks can memory multiple patterns with only one weight matrix. And the association by fuzzy neural networks is completely in accordance with memorized patterns.

\section{PRACTICAL APPLICATION}

\subsection{Introduction of research conditions}

The research of fault diagnosis strongly emphasizes the practical application. With the financial aid of National Natural Science Funds of China, our research group has been co-operating with the oil plant in the research of the online monitoring, fault diagnosis and prediction of rotary machines for several years.

Fig. 5 is a group of photos of the water pump sets of oil plants. (a) is outline of water pump. The green box is motor and the gray box is pump. (b) is gearbox. The red arrow points to vibration sensor. (c) is front bearings of pump. Red arrow points to vibration sensor. There are two sensors on the bearings. One is in the horizon direction and the other is in vertical direction. (d) is the rotor of the water pump It is the key part of the machine. (e) is back bearings and (f) is monitor panel of computer.

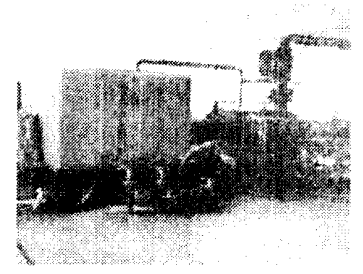

(a) Out line of pump sets

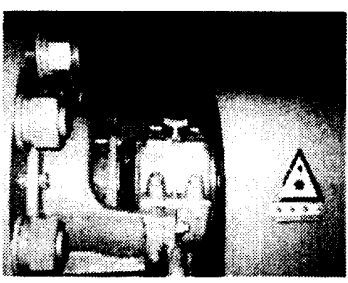

(c) Front bearings

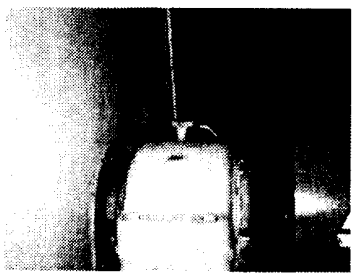

(b) Gear box

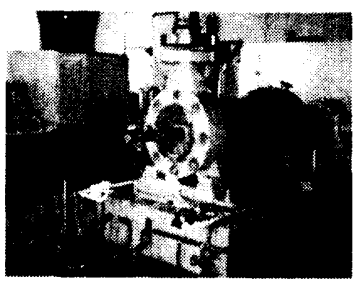

(d) rotor of pump

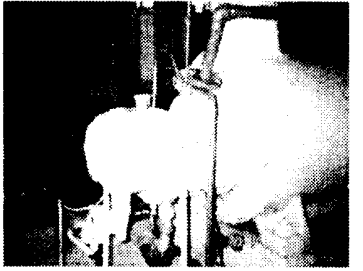

(e) Back bearings

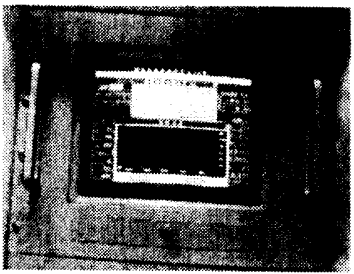

(f) Monitor panel
Fig. 5 Water pump sets (Red arrow points vibration sensor)

To make it clear, Fig. 6 Shows the Structure of the water pump set. The "* " represent the places in which the vibration sensors are installed.

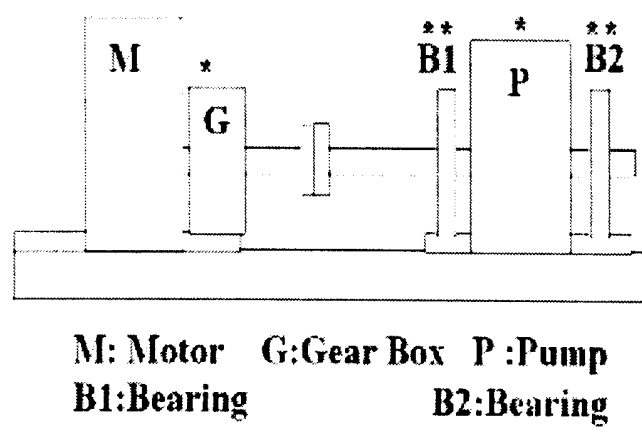

Fig. 6 Brief chart of the water pump set

6 vibration sensors are installed in one pump set. One is on the gearbox. Two are on the bearings in front of the pump. Two are on the bearings behind the pump. one is on the body of the pump.

For install place of vibration sensor, two factors are considered. One is if the place is sensitive to reflect the change of working condition. The other is that convenience to install and maintain the sensors.

\subsection{Fuzzy neural networks for fault diagnosis}

The fault diagnosis system by fuzzy neural networks for water pump sets is the same as the neural networks showed in Fig 4.

The inputs are vibration intensity of six vibration sensors. The fuzzy layer changes the input of vibration intensity into fuzzy value as $0.2,0.4,0.6,0.8,1$ as Fig 2 shows. The outputs are probability in 6 main faults. The severity of fault also fuzzy into 5 class as $0.2,0.4,0.6,0.8,1$. The fuzzy method is very effective to handle practical sample data.

\subsection{Input of fuzzy neural networks}

From 6 vibration sensors, vibration velocity values are measured. However, vibration velocity values are not 
directly used to make fault diagnosis. Instead of vibration velocity values, vibration intensity values are used to make fault diagnosis. Vibration intensity' definition is showed as equation (12):

$$
V_{r m s}=\sqrt{\frac{1}{N \Delta t} \sum_{i=0}^{N-1} V_{i}^{2}(i)}
$$

$\Delta t \quad$ sample interval $(0.166 \mathrm{~ms})$

$\mathrm{N} \quad$ sample number per second (1024/second )

$\mathrm{V}(\mathrm{t})$ vibration velocity $(\mathrm{mm} / \mathrm{s})$

$V_{\text {rms }} \quad$ vibration intensity $(\mathrm{mm} / \mathrm{s})$

Vbration intensity values are normalized from 0 to 1 . The alarm value is set as 1. Fig. 7 show the obsevation of vibration intensity values.

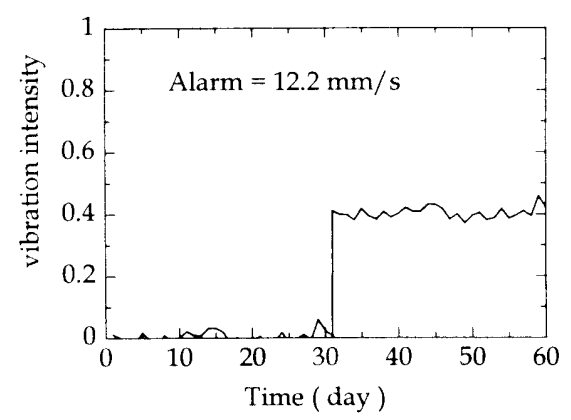

Fig. 7 fuzzy of vibration intensity

With six sensors, six vibration intensity values are obtained as input of fuzzy neural networks. There is input vector $\mathrm{X}$ as equation (13)

$$
\mathrm{X}=[0.2,0.2,0.8,0.8,0.2,0.2]
$$

As Fig 6 shows, $\mathrm{X}$ can expressed as follows:

Vibration intensity of sensor in gearbox is 0.2

Vibration intensity of sensor in pump body is 0.2

Vibration intensity of A sensor of front bearing is 0.8

Vibration intensity of B sensor of front bearing is 0.8

Vibration intensity of A sensor of back bearing is 0.2

Vibration intensity of B sensor of back bearing is 0.2

As Table. 6 shows, six kinds of standard pattern input are used to train the fuzzy neural networks.

Table. 6 Standard patterns for training

\begin{tabular}{|c|c|c|c|c|c|}
\hline $\mathrm{X}_{1}$ & $\mathrm{X}_{2}$ & $\mathrm{X}_{3}$ & $\mathrm{X}_{4}$ & $\mathrm{X}_{5}$ & $\mathrm{X}_{6}$ \\
\hline 0.8 & 0.2 & 0.2 & 0.2 & 0.2 & 0.2 \\
\hline 0.2 & 0.8 & 0.4 & 0.2 & 0.2 & 0.2 \\
\hline 0.4 & 0.2 & 0.8 & 0.6 & 0.2 & 0.2 \\
\hline 0.4 & 0.2 & 0.6 & 0.8 & 0.4 & 0.2 \\
\hline 0.2 & 0.2 & 0.2. & 0.4 & 0.8 & 0.6 \\
\hline 0.2 & 0.2 & 0.2 & 0.2 & 0.4 & 0.8 \\
\hline
\end{tabular}

\subsection{Output of fuzzy neural networks}

In the working condition of water pump set, there are mainly 6 kinds of fault states. A vector of six numbers is used to express the result of fault diagnosis. Every number of the vector expresses probability of state in one of the six main states a. For example, result $\mathrm{Y}$ as equation (14) shows:

$$
Y=[0.8,0.4,0.2,0.2,0.2,0.2]
$$

Represents that

(1) Probability of gearbox fault is 0.8

(2) Probability of misalignment rotor fault is 0.4

(3) Probability of front bearings fault is 0.2

(4) Probability of cracked rotor fault is 0.2

(5) Probability of loosen base fault is 0.2

(6) Probability of back bearings fault bearings is 0.2

When six standard patterns input, correspondingly the output are listed as Table. 7 shows:

Table.7 Output for standard input

\begin{tabular}{|l|l|l|l|l|l|l|}
\hline $\mathrm{Y}_{1}$ & 0.8 & 0.2 & 0.2 & 0.2 & 0.2 & 0.2 \\
\hline $\mathrm{Y}_{2}$ & 0.2 & 0.8 & 0.2 & 0.2 & 0.2 & 0.2 \\
\hline $\mathrm{Y}_{3}$ & 0.2 & 0.2 & 0.8 & 0.2 & 0.2 & 0.2 \\
\hline $\mathrm{Y}_{4}$ & 0.2 & 0.2 & 0.2 & 0.8 & 0.2 & 0.2 \\
\hline $\mathrm{Y}_{5}$ & 0.2 & 0.2 & 0.2 & 0.2 & 0.8 & 0.2 \\
\hline $\mathrm{Y}_{6}$ & 0.2 & 0.2 & 0.2 & 0.2 & 0.2 & 0.8 \\
\hline
\end{tabular}

\subsection{Practical fault diagnosis cases}

The fault diagnosis by fuzzy neural networks is installed in the water pump sets of the oil plant, monitoring the working condition of pump sets all year round without break. The following are two practical cases of fault diagnosis of the water pump station. Both fault diagnosis results were verified to be in accordance with practical faults.

Case 1: The fault diagnosis system indicates the machine is in abnormal working condition, the front bearings are over worn. The input vector from sensors is

$$
\mathrm{X}=[0.2,0.2,0.8,0.6,0.2,0.2]
$$

The associated vector by the fuzzy neural networks is:

$$
\mathrm{Z}=[0.2,0.2,0.8,0.2,0.2,0.2]
$$

Fault diagnosis result is that probability of front bearings fault is 0.8 . The workers checked the front bearings of the pump, verified that some of the rollers of the bearings are over-worn. The diameter of one roller is $0.4 \mathrm{~mm}$ smaller than the normal size.

Case 2: The fault diagnosis system indicates the machine is in abnormal working condition, the gearbox is in abnormal vibration. The input vector from sensors is 


$$
X=[0.6,0.2,0.4,0.2,0.2,0.2]
$$

The associated vector by the fuzzy neural networks is:

$$
\mathrm{Z}=[0.6,0.2,0.2,0.2,0.2,0.2]
$$

Fault diagnosis result is that probability of gearbox is 0.6 . The workers checked the gearbox, verified that the high speed gear has a crack of length of $3 \mathrm{~mm}$.

\section{CONCLUSION}

In this research, the application of the optimized fuzzy neural networks is proposed to make fault diagnosis. The fault diagnosis system by fuzzy neural networks is installed in water pump sets of oil plants. The effectiveness is satisfied. The conclusion are summarized as following:

(1) Fuzzy neural network can memorize fault patterns and associate. It can be used to make accurate fault diagnosis.

(2) Fuzzy neural networks has good tolerance to input sample data. In practical fault diagnosis, sample data are usually very uncertain. Through experiments, it was verified fuzzy neural networks are capable to operate practical data.

Though this research, it can be concluded that to adopt fuzzy logic in neural networks is very effective to make practical fault diagnosis.

\section{REFERENCES}

[1] Xiaoli $\mathrm{Xu}$, Doctor degree thesis, The research of online monitoring and trend predicting of large scale rotary sets (in Chinese), Beijing University of Science And Technology, P36,1998
[2] Watanabe K.,Himmelblau. DM Default Diagnosis in Nonlinear Chemical Processing: Theory. Aiche J,1983,29:243-250

[3] Leonard James A, Kraner Mark A. Radial basis function networks for classing process faults. IEEE Control System Magazine, 1991, 11(3):31-28

[4] K Tanaka, M Sano. Frequency shaping for fuzzy control system with unknow nonlinear plants by a learning method of neural network. Fuzzy Sets and Systems, 1995, 71(1),71-84

[5] Van Way, C. B., Kudva, J. N., and Marantidis, C., Mark N. West. Guidelines for Smart Structural Health Monitoring Systems for Military Aircraft--Lessons Learned from the SSCORE Contract. North American Conference on Smart Structures and Materials, Orlando, Froride 1994,Paper No. 2191-25

[6] Totsos J K. Behaviorist Intelligence and the Scaling Problem. Artificial Intelligence,1995,75(2):135 60

[7]Vincent W.P. et al. Alternative Neural Network Training Methods. IEEE Expert,1995:16 22

[8] Masumi I. Learning of Modular Structured Networks. Artificial Intelligence, 1995,75:51 62

[9] Nelson M. and Herve A.B. Neural Networks for Statistical Recognition of Continuous Speech. Proc. Of the IEEE,1995,83(5):742 770

[10] S. L. Choon, D. A. Dornfeld, S. E. Dreyfus. A Customized Neural Network for Sensor Fusion in On-Line Monitoring of Cutting Tool Wear. Journal of Eng. for Industry, 1995, 117(1):152 159

[11]Buckley J.J.Hayashi Y. Fuzzy Neural Networks: A Survey . Fuzzy Sets and Systems,1994,66(1):1 13

[12] Zhenliang Liu, Fuzzy logic and Neural Networks, Beijing University of Aviation Press, (in Chinese), p208,1995

[13] Kosko B. Fuzzy Associative Memories, FuzzyExpert System, Kandel A, Ed Addison 1987

[14] Kosko B. Competitive Adaptive bi-directional associative memories, Proc.IEEE Int Cof NY 2,P261-268, 1988 